\title{
First patient in the Iranian Registry with novel DOCK2 gene mutation, presenting with skeletal tuberculosis, and review of literature
}

\author{
Niusha Sharifinejad ${ }^{1}$, Homa Sadri $^{1,4^{*}}$, Arash Kalantari ${ }^{2}$, Samaneh Delavari ${ }^{3}$, Amirhosein Noohi ${ }^{4}$, \\ Yasaman Aminpour ${ }^{4}$, Araz Sabzevari ${ }^{5}$ and Gholamreza Azizi ${ }^{1,3^{*}}$
}

\begin{abstract}
Background: Dedicator of cytokinesis 2 (DOCK2) deficiency is an inborn error of immunity characterized by cellular and humoral immunological abnormalities leading to early-onset infections.

Case presentation: We reported a novel case of a 27 months old girl presenting with recurrent pneumonia and a history of skeletal tuberculosis at the age of 19-month-old. Her immunological workup revealed persistent lymphopenia and low CD4 + T cell count along with elevated levels of CD19+, CD20 +, CD16 +, and CD56+ cells. Furthermore, she had a high level of immunoglobulin (Ig) E and a slightly reduced IgM level with a non-protective antibody titer against diphtheria. The whole-exome sequencing (WES) analysis identified a homozygous frameshift deletion mutation (c.1512delG, p.1505Sfs*28) in exon 16 of the DOCK2 gene. We also conducted electronic searches in PubMed, Web of Science, and Scopus databases and reviewed the articles reporting patients with DOCK2 deficiency. The literature search yielded 14 DOCK2-deficient patients suffering from both cellular and humoral immune defects leading to early-onset infections, particularly human herpesvirus (HHV) infection.
\end{abstract}

Conclusion: DOCK2 deficiency should be considered in the context of severe or unusual early-onset infections, especially HHV infections, in a patient with a probable clinical diagnosis of combined immunodeficiency. We also recommended that DOCK2-deficient patients might benefit from T-cell receptor excision circle (TREC) assay as part of the routine newborn screening program.

Keywords: Primary Immunodeficiency, Dedicator of cytokinesis 2 deficiency, DOCK2 deficiency, Combined immunodeficiency, CID

\footnotetext{
*Correspondence: Homasadri7@gmail.com; azizi@abzums.ac.ir

${ }^{1}$ Non-Communicable Diseases Research Center, Alborz University of Medical Sciences, Karaj, Iran

${ }^{4}$ Department of Pediatrics, Imam Ali Hospital, Alborz University of Medical Sciences, Karaj, Iran

Full list of author information is available at the end of the article
}

\begin{abstract}
Introduction
Dedicator of cytokinesis 2 (DOCK2) is a subfamily of guanine exchange factors required for the activation of intercellular GTPases and subsequent release of adenosine triphosphate (ATP) in response to various stimuli [1]. DOCK2, specifically, expresses on immune cells and promotes the activation and migration of $\mathrm{B}$ cell and $\mathrm{T}$ cell lymphocytes. Besides, it promotes the cytotoxicity and degranulation of natural killer (NK) cells
\end{abstract} permits use, sharing, adaptation, distribution and reproduction in any medium or format, as long as you give appropriate credit to the original author(s) and the source, provide a link to the Creative Commons licence, and indicate if changes were made. The images or other third party material in this article are included in the article's Creative Commons licence, unless indicated otherwise in a credit line to the material. If material is not included in the article's Creative Commons licence and your intended use is not permitted by statutory regulation or exceeds the permitted use, you will need to obtain permission directly from the copyright holder. To view a copy of this licence, visit http://creativecommons.org/licenses/by/4.0/. The Creative Commons Public Domain Dedication waiver (http://creativeco mmons.org/publicdomain/zero/1.0/) applies to the data made available in this article, unless otherwise stated in a credit line to the data. 
and the thymic development of NKT cells. Additionally, DOCK2 is reported to mediate the production of reactive oxygen species (ROS) and chemotaxis in neutrophils [2].

Biallelic loss-of-function mutations in the DOCK2 gene are associated with clinical features of combined immunodeficiency (CID) according to the primary immunodeficiency (PID) treatment consortium (PIDTC) $[3,4]$. Different DOCK2 mutations resulted in absent or significantly reduced levels of DOCK2 protein expression that precede a chain of immunological defects including CD4+ and CD8 $+\mathrm{T}$ cells lymphopenia with reduced $\mathrm{T}$ cell receptor excision circles (TRECs), diminished phytohemagglutinin (PHA)-induced $\mathrm{T}$ cell proliferation, impaired NK cell function, decrease B cell counts, and defective antibody responses in DOCK2deficient patients [3]. These immunological disturbances commence early-onset and severe infections in patients harboring DOCK2 mutations that often lead them to death [5]. Hematopoietic stem cell transplantation (HSCT) is currently the only curative option for DOCK2deficient patients [3].

Here, we report a 27 months old girl who presented with severe infection and carried a novel homozygous frameshift deletion c.1512delG (p.I505Sfs*28) mutation at exon 16 of the DOCK2 gene. We have also reviewed the data of previously reported patients with DOCK2 deficiency.

\section{Patient and methods}

The demographic data, medical history, and physical examination of the patient were obtained through direct interviews and examining the patient's clinical record in the Imam-Ali Karaj Hospital affiliated to Alborz University of Medical Sciences, using national consensus on diagnosis and management guidelines for PID [6]. Written informed consent was obtained from the parents, following the principles of the ethics committee of the Alborz University of Medical Sciences. Basic hematological, biochemistry, and immunological investigations including enumeration of lymphocyte subsets: T- and B-cells subsets (assessed using flow cytometry analysis), serum levels of immunoglobulins, complements, and antibodies (assessed using nephelometry and enzyme-linked immunosorbent assay).

Secondary causes of lymphopenia were excluded by history taking and laboratory tests, and no sign of drug or disease-related causes was detected. Clinical diagnosis of CID has been established according to the criteria of the European Society for Immunodeficiencies [7]. The whole peripheral blood sample was used to extract genomic DNA. The whole-exome sequencing (WES) and the confirmatory Sanger sequencing were performed according to a previously published pipeline $[8,9]$.

The literature searches for reported DOCK2-deficient patients were conducted in PubMed, Web of Science, and Scopus, using the following keywords: DOCK2 deficiency and Dedicator of cytokinesis 2 deficiency. The articles were primarily screened based on the title and abstract to exclude immaterial studies and then the full-text manuscripts of the included studies were assessed to determine their eligibility criteria (written in English, conducted on human subjects, reporting at least one patient with DOCK2 deficiency diagnosis). The descriptive section is subsequently developed based on this information.

\section{Case presentation}

The patient was a 26-month-old girl born to first-degree consanguineous Iranian/Turkish parents. She was born at term gestational age with $3200 \mathrm{~g}$ weight and underwent routine age-matched vaccination in Iran including BCG vaccine at birth, Polio, Hepatitis B, Diphtheria, Tetanus, Haemophilus Influenza type B, Pertussis, and MMR (mumps, measles, rubella) until 18 months old, without remarkable adverse event. She had an older sister who died at the age of 1.5 years with diarrhea as the main clinical symptom (Fig. 1). At 18 months of age, she was hospitalized due to pneumonia and otitis media with perforated tympanic membrane and pus discharge. By that time, her growth and developmental state were age-appropriate and she had not experienced prior hospitalization or considerable infection. Only a few days after her discharge, she developed right thigh swelling, tenderness, and heat, unable of weight-bearing.

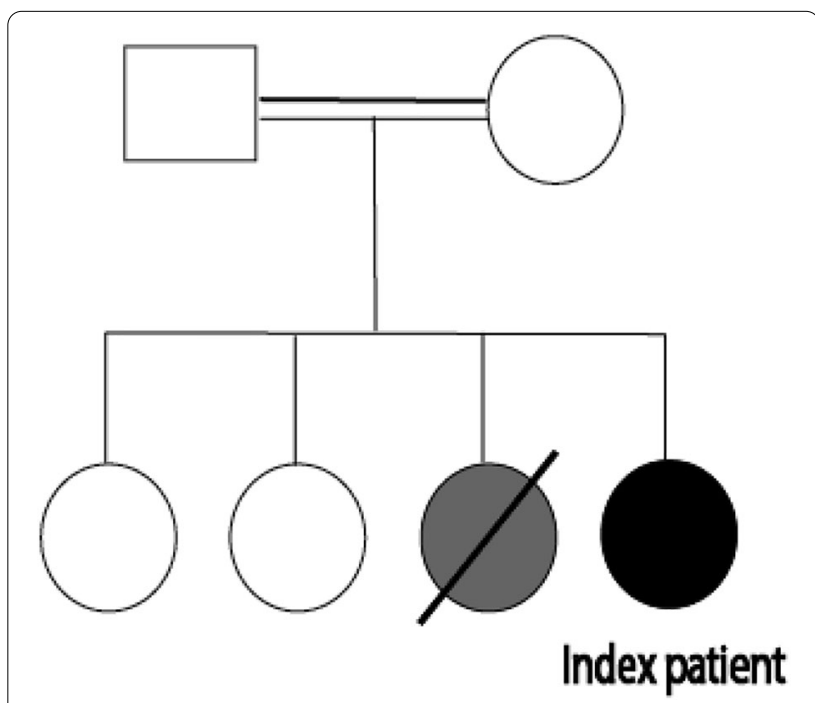

Fig. 1 The family pedigree of the patient with DOCK2 deficiency 
Therefore, she was admitted once more for suspected acute osteomyelitis. The right femur X-ray showed evidence of osteomyelitis (Fig. 2). Later, she underwent an open biopsy and the mycobacterium tuberculosis (MTB) DNA complex was detected in the tissue sections obtained from her right femur, using Xpert MTB/RIF Ultra assay which is a rapid and accurate diagnostic tool, especially in extra-pulmonary tuberculosis [10]. Plus, the smear of skeletal canal fluid specimen confirmed the presence of many acid-fast positive bacilli. Meantime, chest radiographs of the patient and her parents were evaluated and although her parents had no lesion compatible with pulmonary $\mathrm{TB}$, the patient's X-ray showed round density in the middle lobe of the right lung. They had no contact history of TB. She was treated with Isoniazid, Rifampin, Ethambutol, Pyrazinamide, and vitamin $\mathrm{B} 6$ for 9 months with the diagnosis of skeletal tuberculosis. The patient's unusual infection with an uncommon microorganism prompted an immunological workup to detect the possible underlying immunodeficiency (Table 1). Based on her clinical and initial laboratory data, she was recommended for a

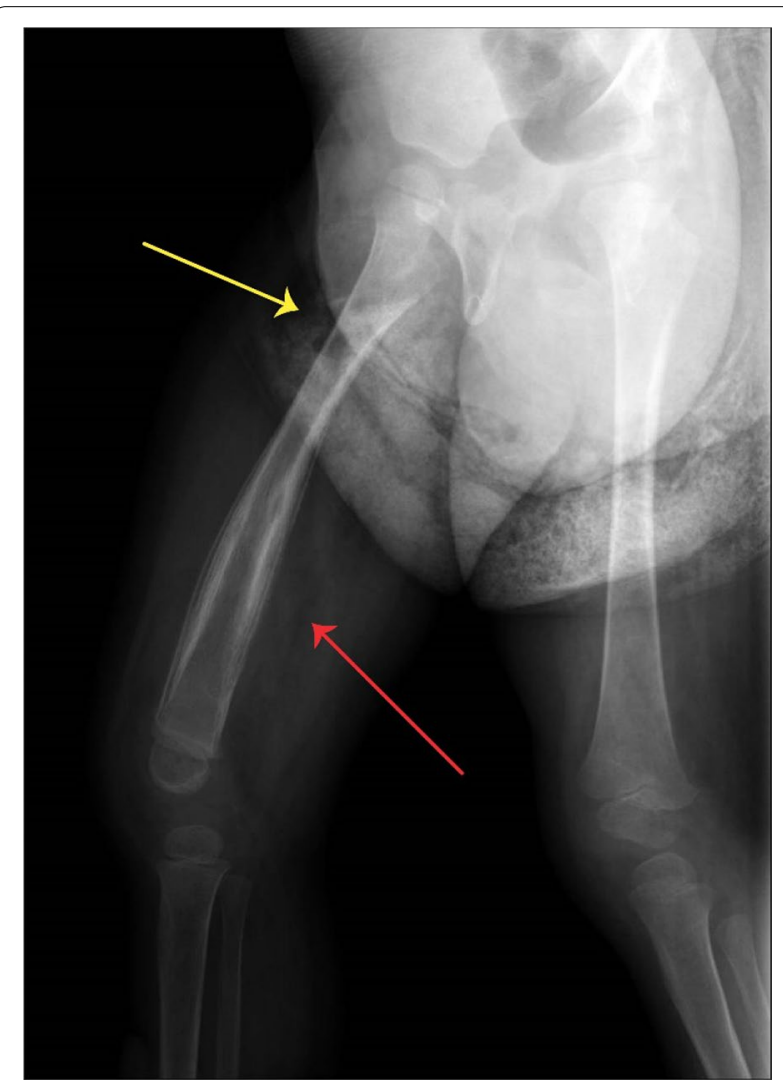

Fig. 2 The X-ray of the patient shows signs of periosteal reaction in the diaphysis (red arrow) and a lucent area with sclerotic borders in the metaphysis of right femur (yellow arrow) thorough evaluation, but her parents refused further investigation.

A few months later, she was referred to our hospital with fever, cough, and shortness of breath and eventually admitted to the pediatric intensive care unit (PICU) due to severe tachypnea and respiratory distress with the diagnosis of severe pneumonia. During her admission, she received empirical broad-spectrum antibiotics with full coverage of bacteria, viruses, and fungi. Nonetheless, no pathogenic microorganism was isolated from the patient and despite receiving the standard treatment, her respiratory condition worsened and she got intubated.

Her inadequate response to standard treatment led to afresh immunological workup. The laboratory results revealed anemia, lymphopenia along with decreased $\mathrm{CD} 4+$ count, and CD4/CD8 ratio similar to her previous records (Table 1). She also had increased CD19+, $\mathrm{CD} 20+, \mathrm{CD} 16+$, and $\mathrm{CD} 56+$ cell counts. Her antidiphtheria antibody titer was beneath the protective level despite the age-matched vaccination. She had hyper immunoglobulin (Ig) E and a slightly reduced IgM level (Table 1). Based on her results, a probable CID was suspected and genetic analysis was performed on whole blood samples using WES. The WES analysis and Sanger sequencing revealed a homozygous frameshift deletion mutation c.1512delG (p.I505Sfs*28) at exon 16 in the DOCK2 gene loci.

Meanwhile, her serum creatinine and urea level increased significantly $(2.5$ and $248.5 \mathrm{mg} / \mathrm{dL}$, respectively) and she underwent peritoneal dialysis. During her admission, she suffered from pneumothorax and few episodes of seizure as well. Unfortunately, she was deceased at the age of 27-month-old due to multiorgan failure.

\section{Review of literature}

So far, 14 DOCK2-deficient cases ( 8 males, 4 females, and 2 unknown gender) were reported in six different studies from various ethnicities. Consanguinity and positive family history of immunodeficiency were reported in $81.8 \%$ (9 out of 11 cases) and $44.4 \%$ (4 out of 9 cases) of patients, respectively. All patients with available data developed infection as their first manifestation of immunodeficiency within the first months of birth. Half of the patients (6 of 12) also presented with infections caused by different members of the human herpesvirus family (HHV) including HHV6, varicella, and Cytomegalovirus (CMV). Fifty-seven percentage (8 of 12) of patients had lymphopenia. The major immunological abnormalities in lymphocyte subsets were; low CD3+ in $64.2 \%$ (9 of 14), low CD4+ in 78.6\% (11 of 14), low CD8 + in 50\% (7 of 14), low CD19+ in 50\% (7 of 14), and low NK counts in $50 \%$ (7 of 14). 
Table 1 Immunologic work-up of the DOCK2 deficient-patient

\begin{tabular}{|c|c|c|c|}
\hline Parameters & 19 months old & 26 months old & Normal ranges \\
\hline WBC $\times 10^{3}($ cell $/ \mu \mathrm{L})$ & 25.5 & 6.7 & $5.5-15.5$ \\
\hline Lymphocytes (cell/ $\mu \mathrm{L}$ ) & 11,700 & 1641 & $3873-6141$ \\
\hline Hemmoglobin (g/dL) & 9.5 & 9.8 & $10.9-15$ \\
\hline CD3 + T cells (cell/ $\mu \mathrm{L})$ & 6470 & 1709 & $1578-3707$ \\
\hline CD4+T cells (cell/ $\mu \mathrm{L})$ & 322 & 34 & $870-2144$ \\
\hline CD8+T cells (cell/ $\mu \mathrm{L})$ & 2697 & 581 & $472-1107$ \\
\hline CD4/CD8 Ratio & 0.16 & 0.058 & $0.9-3.7$ \\
\hline 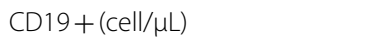 & 2597 & 1900 & $434-1274$ \\
\hline 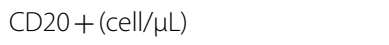 & ND & 1900 & $124-1665$ \\
\hline 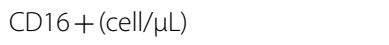 & 1111 & 1178 & 78-703 \\
\hline CD56+ (cell/ $\mu \mathrm{L})$ & 1743 & 874 & $45-555$ \\
\hline CD16+56 (cell/uL) & 760 & 380 & $155-565$ \\
\hline $\operatorname{lgG}(\mathrm{mg} / \mathrm{dL})$ & ND & 511 & $424-1051$ \\
\hline $\operatorname{lgM}(\mathrm{mg} / \mathrm{dL})$ & 79 & 41 & $48-168$ \\
\hline $\lg A(m g / d L)$ & 72 & 85.1 & $14-123$ \\
\hline $\lg \mathrm{E}(\mathrm{IU} / \mathrm{mL})$ & 471 & 400 & $<100$ \\
\hline C3 (mg/mL) & 113 & ND & $84-174$ \\
\hline $\mathrm{C} 4(\mathrm{mg} / \mathrm{mL})$ & 27 & ND & $12-40$ \\
\hline CH50 (IU/mL) & $>90$ & 116 & $70-150$ \\
\hline MFI/PMA ox-DHR (\%) & $40.2 \%$ & ND & $>30 \%$ \\
\hline HIV 1,2 antigen/ antibody (S/CO) & ND & 0.03 & $<0.9$ \\
\hline Anti-tetanus antibody (IU/mL) & ND & 0.19 & $>0.1$ \\
\hline Anti-diphtheria antibody (IU/mL) & ND & 0.03 & $>0.1$ \\
\hline
\end{tabular}

ND not determined, Ig immunoglobulin, WBC white blood cell, C3 complement component 3, C4 complement component 4, CH50 total hemolytic complement, MFI/ $P M A$ ox-DHR mean fluorescence intensity/phorbol myristate acetate oxy dihydrorhodamine, HIV 1,2 human immunodeficiency virus 1,2

Abnormal value are indicates in bold

IgM levels were decreased in 7 cases (50\%) and increased in 3 cases (21.4\%). Elevated/or reduced IgA levels were present in 3 cases (21.4\%). IgG levels were mainly normal among the patients (78.6\%). Nine patients showed decreased T-cell response to PHA, and of those, six patients had the SCID response range [11]. All of the cases that were tested for TREC (4 cases) had decreased TREC levels. Poor response to vaccination and absent antibody responses to infectious agents were also reported in some cases. HSCT was performed as the main treatment in $77.8 \%$ (7 of 9) of patients resulting in achieved engraftment in 5 (71.4\%) patients. Further information is available in Table 2.

\section{Discussion}

In this study, we reported a 27-month-old girl presenting with recurrent, severe, early-onset infections. The laboratory data revealed defective cellular and humoral immune systems and the patient was found to harbor a novel homozygous frameshift deletion mutation c.1512delG (p.I505Sfs*28) at exon16 of the DOCK2 gene. Similar to the immunological results of patients reported by Dobbs et al. and Moens et al. [3, 12], our patient had lymphopenia along with decreased CD4+ cell count and IgM level. However, in contrast to our patient's results, these studies also described decreased CD3 + and CD8 + cell counts as well as defective antibody responses in patients with DOCK2 deficiency $[3,12]$. Of note, the persistent lymphopenia observed in DOCK2-deficient patients could be due to the recurrent infections that occurred in these cases, which is more prominent in our patient who manifested with skeletal tuberculosis, an unusual infection that has not been previously reported. In contrast, the index patient in Moens et al.'s study was reported to have lymphopenia and reduced TREC level at birth and prior to infection [12]. Therefore, more studies are required to elucidate whether lymphopenia is inherent to DOCK2 deficiency. Additionally, our patients received age-matched vaccination including $B C G$, and did not represent any adverse reaction.

Dobbs et al. were the first study that described DOCK2-deficient patients with three out of the five patients developing HHV infections [3]. Later, Alizadeh et al. proposed a hypothesis based on the 


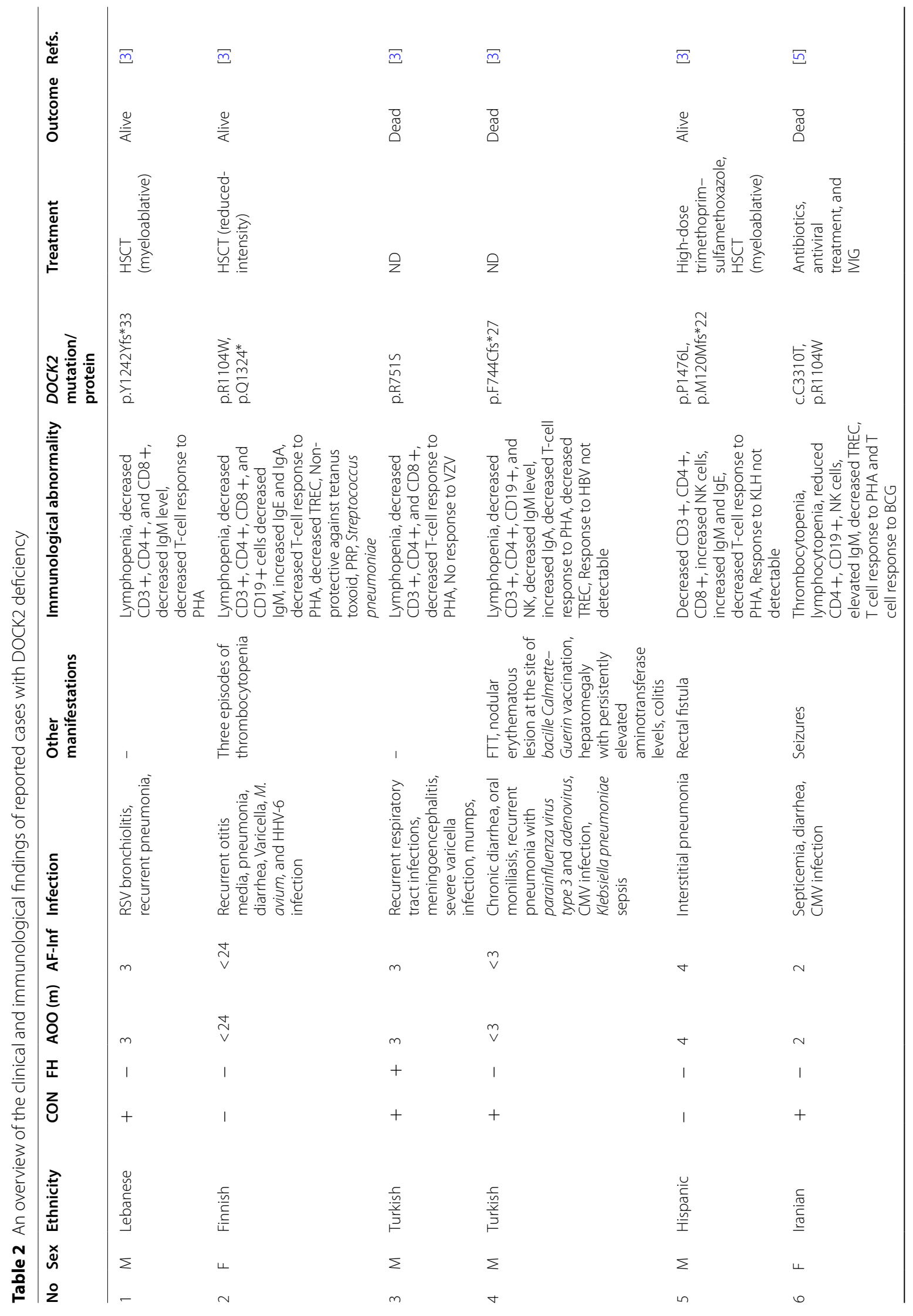




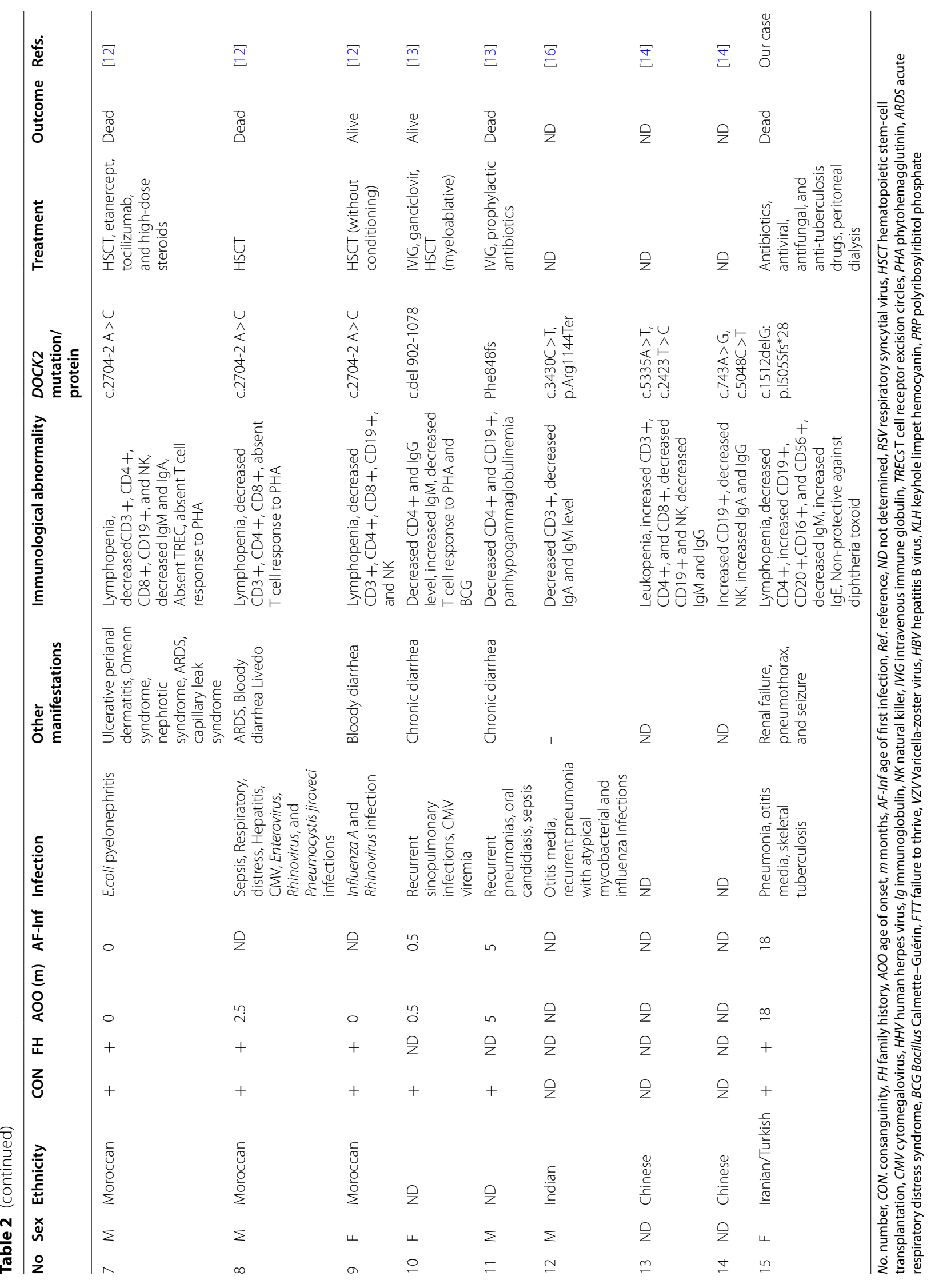


results of their patient and Dobbs et al.'s, indicating a probable association between DOCK2 deficiency and susceptibility to various HHV infections [5] which was subsequently strengthened by the results of other studies [12, 13]. Even so, no sign of HHV-related infections was detected in our case. We also observed elevated IgE level in our patient that was only expressed previously by Dobbs $K$ et al. [3]. Unlike our patient's laboratory data, most of the DOCK2-deficient patients had decreased CD19+ cell counts. However, a recent study of a Chinese patient also reported an increased CD19+ cell count [14].

We found a total of 14 DOCK2-deficient patients skewing toward male patients $(57.1 \%)$ in the published literature. Positive family history of immunodeficiency and parental consanguinity were important factors in the patients that are affirmative according to the autosomal recessive inheritance of DOCK2 deficiency [3]. The prevalent immunological abnormalities among reported patients were as follows: low $\mathrm{CD} 3+$, low $\mathrm{CD} 4+$, low $\mathrm{CD} 8+$, low $\mathrm{CD} 19+$, and low NK cell counts along with reduced IgM and normal IgG levels. Decreased T-cell responses to PHA were also present in the range defined for SCID [11]. Given all cases with available data had low TREC levels and the arguable evidence of lymphopenia adherence to this disease, DOCK2 deficiency could be recognizable through newborn SCID screening programs [15]. Unfortunately, we were not able to determine the T-cell response to PHA and TREC levels of our patient due to her rather rapid clinical deterioration and death.

\section{Conclusion}

In summary, DOCK2 deficiency should be contemplated in the context of severe or unusual early-onset infections, especially HHV infections, accompanied by laboratory data indicating both cellular and humoral defects. Decreased CD4 $+\mathrm{T}$ cell count was the most prevalent immunological abnormality detected in these patients. The role of the newborn TREC screening program in detecting suspected patients with DOCK2 deficiency needs to be clarified in the upcoming studies. Further investigations are required to discover the possible associations between this genetic defect and the laboratory/or clinical features of the disease.

\footnotetext{
Abbreviations

DOCK2: Dedicator of cytokinesis 2; Ig: Immunoglobulin; HHV: Human herpes virus; NK: Natural killer; ATP: Adenosine triphosphate; ROS: Reactive oxygen species; CID: Combined immunodeficiency; PIDTC: Primary immunodeficiency treatment consortium; MTB: Mycobacterium tuberculosis; TRECs: T cell receptor excision circles; PHA: Phytohemagglutinin; HSCT: Hematopoietic stem cell transplantation; SCID: Severe combined immunodeficiency;
}

WES: Whole-exome sequencing; PICU: Pediatric intensive care unit; CMV: Cytomegalovirus.

\section{Acknowledgements}

Authors are grateful to patient and her family who accepted to be involved in the study. A special acknowledgement to Dr. Mohammad Shahrooei, [Specialized Immunology Laboratory of Dr. Shahrooei, Sina Medical Complex, Ahvaz, Iran], for his assistance in molecular diagnosis of patient.

\section{Authors' contributions}

NS; the acquisition of data, analysis and interpretation of data, and have drafted the work or substantively revised it, HS; the acquisition of data, have made substantial contributions to the conception, design of the work, AK; the acquisition of data, have made substantial contributions to the conception, substantively revised the work, SD; the acquisition and interpretation of data, AS; have drafted the work or substantively revised it, GA; interpretation of data, design of the work, drafted the work or substantively revised it. All authors read and approved the final manuscript.

\section{Funding}

This work was supported by the vice chancellor for research, Alborz University of Medical Sciences under Grant Number 99-4032.

\section{Availability of data and materials}

All data generated or analyzed during this study are included in this published article.

\section{Declarations}

Ethics approval and consent to participate

Not applicable.

\section{Consent for publication}

Informed consent was obtained from the parents of the patient prior to being included in the study.

\section{Competing interests}

The authors declare that they have no competing interests.

\section{Author details}

${ }^{1}$ Non-Communicable Diseases Research Center, Alborz University of Medical Sciences, Karaj, Iran. ${ }^{2}$ Department of Immunology and Allergy, Imam Khomeini Hospital, Tehran University of Medical Sciences, Tehran, Iran. ${ }^{3}$ Research Center for Immunodeficiencies, Pediatrics Center of Excellence, Children's Medical Center, Tehran University of Medical Science, Tehran, Iran. ${ }^{4}$ Department of Pediatrics, Imam Ali Hospital, Alborz University of Medical Sciences, Karaj, Iran. ${ }^{5}$ CinnaGen Medical Biotechnology Research Center, Alborz University of Medical Sciences, Karaj, Iran.

Received: 22 April 2021 Accepted: 19 November 2021

Published online: 06 December 2021

\section{References}

1. Nishihara H, Kobayashi S, Hashimoto Y, Ohba F, Mochizuki N, Kurata T, Nagashima K, Matsuda M. Non-adherent cell-specific expression of DOCK2, a member of the human CDM-family proteins. Biochim Biophys Acta. 1999;1452:179-87.

2. Chen Y, Meng F, Wang B, He L, Liu Y, Liu Z. Dock2 in the development of inflammation and cancer. Eur J Immunol. 2018:48:915-22.

3. Dobbs K, Domínguez Conde C, Zhang S-Y, Parolini S, Audry M, Chou J, Haapaniemi E, Keles S, Bilic I, Okada S, et al. Inherited DOCK2 deficiency in patients with early-onset invasive infections. N Engl J Med. 2015;372:2409-22.

4. Shearer WT, Dunn E, Notarangelo LD, Dvorak CC, Puck JM, Logan BR, Griffith LM, Kohn DB, O'Reilly RJ, Fleisher TA, et al. Establishing diagnostic criteria for severe combined immunodeficiency disease (SCID), leaky SCID, and Omenn syndrome: the primary immune 
deficiency treatment consortium experience. J Allergy Clin Immunol. 2014;133:1092-8.

5. Alizadeh Z, Mazinani M, Shakerian L, Nabavi M, Fazlollahi MR. DOCK2 deficiency in a patient with hyper IgM phenotype. J Clin Immunol. 2018;38:10-2.

6. Abolhassani H, Tavakol M, Chavoshzadeh Z, Mahdaviani SA, Momen T, Yazdani R, Azizi G, Movahedi M, Hamidieh AA, Behniafard NJI. National consensus on diagnosis and management guidelines for primary immunodeficiency. Immunol Genet J. 2019:2:1-21.

7. Seidel MG, Kindle G, Gathmann B, Quinti I, Buckland M, van Montfrans J, Scheible R, Rusch S, Gasteiger LM, Grimbacher B, et al. The European society for immunodeficiencies (ESID) registry working definitions for the clinical diagnosis of inborn errors of immunity. J Allergy Clin Immunol Pract. 2019;7:1763-70.

8. Alkhairy OK, Abolhassani H, Rezaei N, Fang M, Andersen KK Chavoshzadeh Z, Mohammadzadeh I, El-Rajab MA, Massaad M, Chou J, et al. Spectrum of phenotypes associated with mutations in LRBA. J Clin Immunol. 2016;36:33-45.

9. Fang M, Abolhassani H, Lim CK, Zhang J, Hammarstrom L. Next generation sequencing data analysis in primary immunodeficiency disorders_future directions. J Clin Immunol. 2016;36(Suppl 1):68-75.

10. Hoel IM, Syre H, Skarstein I, Mustafa T. Xpert MTB/RIF ultra for rapid diagnosis of extrapulmonary tuberculosis in a high-income lowtuberculosis prevalence setting. Sci Rep. 2020;10:13959.

11. Alsalamah $M$, Vong $L$, Cimpean L, Dadi H. Establishing reference ranges for lymphocyte proliferation responses to phytohemagglutinin in patients with T cell dysfunction. LymphoSign J. 2019;6:26-30.

12. Moens L, Gouwy M, Bosch B, Pastukhov O, Nieto-Patlàn A, Siler U, Bucciol G, Mekahli D, Vermeulen F, Desmet L, et al. Human DOCK2 deficiency: report of a novel mutation and evidence for neutrophil dysfunction. J Clin Immunol. 2019:39:298-308.

13. Alosaimi MF, Shendi H, Beano A, Stafstrom K, El Hawary R, Meshaal S, Galal N, Pai SY, El-Marsafy A, Geha RS, Chou J. T-cell mitochondrial dysfunction and lymphopenia in DOCK2-deficient patients. J Allergy Clin Immunol. 2019:144:306.

14. Yang L, Jing YK, Wang WJ, Ying WJ, Lin L, Chang J, Luo L, Kang DQ, Jiang PP, Liu J, et al. DOCK2 couples with LEF-1 to regulate B cell metabolism and memory response. Biochem Biophys Res Commun. 2020;529:296-302.

15. van der Burg M, Mahlaoui N, Gaspar HB, Pai S-Y. Universal newborn screening for severe combined immunodeficiency (SCID). Front Pediatr. 2019. https://doi.org/10.3389/fped.2019.00373.

16. Arunachalam AK, Maddali M, Aboobacker FN, Korula A, George B, Mathews V, Edison ES. Primary immunodeficiencies in india: molecular diagnosis and the role of next-generation sequencing. J Clin Immunol. 2021;41:393-413.

\section{Publisher's Note}

Springer Nature remains neutral with regard to jurisdictional claims in published maps and institutional affiliations.

Ready to submit your research? Choose BMC and benefit from:

- fast, convenient online submission

- thorough peer review by experienced researchers in your field

- rapid publication on acceptance

- support for research data, including large and complex data types

- gold Open Access which fosters wider collaboration and increased citations

- maximum visibility for your research: over 100M website views per year

At BMC, research is always in progress.

Learn more biomedcentral.com/submissions 